\title{
Enrichment analysis of differentially expressed genes in chronic heart failure
}

\author{
Xiaoli Fang ${ }^{1 \#}$, Xue Zeng ${ }^{2 \#}$, Miao Wang ${ }^{1}$, Liuqiong Qin ${ }^{2}$, Chuanhui Tan ${ }^{3}$, Jiutao Wu ${ }^{3}$ \\ ${ }^{1}$ Department of Cardiology, Hainan General Hospital, Hainan Affiliated Hospital of Hainan Medical University, Haikou, China; ${ }^{2}$ Department of \\ Internal Medicine, Ding'an Traditional Chinese Medicine Hospital, Ding'an, China; ${ }^{3}$ Department of Internal Medicine-Cardiovascular, Ding'an \\ People's Hospital, Ding'an, China \\ Contributions: (I) Conception and design: X Fang, X Zeng; (II) Administrative support: M Wang; (III) Provision of study materials or patients: X \\ Zeng, J Wu; (IV) Collection and assembly of data: All authors; (V) Data analysis and interpretation: X Fang, J Wu; (VI) Manuscript writing: All \\ authors; (VII) Final approval of manuscript: All authors. \\ \#These authors contributed equally to this work. \\ Correspondence to: Jiutao Wu. Department of Internal Medicine-Cardiovascular, Ding'an People's Hospital, 443 Jianlong Avenue, Dingcheng Town, \\ Ding'an 571200, China. Email: wujiutao1236@163.com.
}

Background The study explores the differentially expressed genes in the heart tissue of patients with chronic heart failure (CHF) and normal heart tissue, thus providing information for further research on the pathogenesis of $\mathrm{CHF}$.

Methods: The Gene Expression Omnibus (GEO) database was used to download the whole transcriptome sequencing results of CHF patients (GSE2656, n=49). Transcriptome sequencing results of 44 normal left ventricular tissues were randomly screened and downloaded using the Genotype-Tissue Expression (GTEX) database $(n=44)$. We explored the differentially expressed genes between CHF tissue and normal heart tissue. Gene Ontology (GO) functional enrichment analysis and Kyoto Encyclopedia of Genes and Genomes (KEGG) signaling pathway enrichment analysis were performed for differentially expressed genes. Growth hormone-releasing hormone $(\mathrm{GHRH})$ was used as a representative differential gene for serological sample verification by the enzyme linked immunosorbent assay (ELISA).

Results: A total of 902 differentially expressed genes between CHF and normal heart tissues were screened, including 354 up-regulated genes and 548 down-regulated genes. GO enrichment analysis showed that the differentially expressed genes were significantly enriched in the extracellular and sequence-specific DNA binding domains. KEGG enrichment demonstrated that the differential genes were enriched in neuroactive ligand-receptor interaction, the calcium signaling pathway, vascular smooth muscle contraction, and other signaling pathways. ELISA results showed that the expression level of GHRH in patients with heart failure was significantly higher than that in healthy subjects $(\mathrm{P}<0.05)$.

Conclusions: A total of 902 differentially expressed genes were found in CHF tissues compared with normal heart tissues. Signaling pathways such as neuroactive ligand-receptor interaction, the calcium ion signaling pathway, and vascular smooth muscle contraction may be related to the pathogenesis of CHF.

Keywords: Chronic heart failure (CHF); transcriptome; enrichment analysis; public database

Submitted Jun 18, 2021. Accepted for publication Jul 26, 2021.

doi: 10.21037/apm-21-1854

View this article at: https://dx.doi.org/10.21037/apm-21-1854 


\section{Introduction}

Chronic heart failure (CHF) is myocardial damage caused by myocardial infarction, cardiomyopathy, hemodynamic overload, inflammation and other reasons. It causes changes in the structure and function of the myocardium, which ultimately leads to hypofunction of pumping or filling of the ventricles. The incidence of CHF has been increasing in recent years, and is often accompanied by a high mortality rate (1-4). Treating CHF involves a complex treatment plan, high costs, and long-term follow-up $(5,6)$. The molecular mechanisms of CHF are complicated and unclear, involving various genetic and environmental factors. Genome-wide gene expression profiling is based on whole-transcriptome sequencing. It is a widely used approach to discover new potential biomarkers, diagnose and predict disease severity, construct a diagnostic evaluation model or prognostic evaluation model, and explore new drug treatment targets (7-9). Transcriptome analysis has been successfully applied to many complex diseases, including malignant tumors, rheumatoid arthritis, and cardiovascular disease (10-12).

In recent years, with the rise of second-generation gene sequencing technology, more chronic heart failure biomarkers have been discovered and verified. A study has identified 89 differentially expressed genes in chronic heart failure tissues (13). These differential genes are related to the extracellular matrix and bioadhesion signaling pathways (13). The study also found that 51 differentially expressed genes encode interacting proteins. A total of 11 key genes including CTGF, POSTN, Corin, FIGF and their related transcription factors were identified (13). However, most of the human genome research on CHF is limited by the lack of clinical samples in patients with advanced CHF. This study explored the differentially expressed genes in heart tissues of patients with CHF and normal heart tissues based on public databases (13). We carried out enrichment analysis to provide information for further research on the pathogenesis of CHF. We present the following article in accordance with the STREGA reporting checklist (available at https://dx.doi. org/10.21037/apm-21-1854).

\section{Methods}

\section{Data resources}

The Gene Expression Omnibus (GEO) database was used to download the full transcriptome sequencing results
(GSE2656, n=49) of myocardial tissues from patients with CHF. The full transcriptome sequencing results of 44 normal left ventricular tissues $(n=44)$ were randomly selected and downloaded from the Genotype-Tissue Expression (GTEX) database. The whole transcriptome data of normal heart tissue and CHF tissue were combined into the same data matrix. The data was batch-corrected to remove the effect of time, temperature, and method, among other factors. Logarithmic transformation of the data matrix was performed after correction and normalization.

\section{Screening of differential genes}

The mRNAs in the data matrix were extracted and analyzed by R software (V.3.5.1) and the edger package. The fold change (FC) was calculated and recorded as $\log _{2} \mathrm{FC}$. The formula was $\log _{2} \mathrm{FC}=\log _{2}$ (CHF tissue/ normal heart tissue). The screening conditions for differentially expressed genes were as follows: (I) $\left|\log _{2} \mathrm{FC}\right|$ $>2$; (II) false discovery rate (FDR) was determined by $\mathrm{P}<0.05$ after correction.

\section{Gene Ontology (GO) analysis}

The database for annotation, visualization, and integrated discovery (DAVID) was used to analyze the GO functions of differentially expressed genes in CHF tissues compared with normal heart tissues. FDR $<0.05$ was used as the selection condition. The Goplot $\mathrm{R}$ package was used to visualize the enrichment results.

\section{Kyoto Encyclopedia of Genes and Genomes (KEGG) signaling pathway enrichment analysis}

The KEGG signaling pathway database was used to analyze the signaling pathway enrichment of differentially expressed genes in CHF tissue compared with normal heart tissue. FDR $<0.05$ was used as the screening condition, and the GOplot $\mathrm{R}$ package was used to visualize the signaling pathway enrichment results.

\section{Differential gene verification}

This study screened representative differential genes for clinical serological sample verification. Select 30 patients with chronic heart failure and 30 healthy subjects in Hainan General Hospital, Hainan Affiliated Hospital of Hainan Medical University to collect venous blood samples. 
Table 1 The top 20 differentially expressed genes with the largest $\mid \log _{2} \mathrm{FCl}$ values

\begin{tabular}{|c|c|c|}
\hline ID & $\log F C$ & FDR \\
\hline GUCA1C & -7.085 & $<0.001$ \\
\hline TMPRSS11B & -6.728 & $<0.001$ \\
\hline AADACL3 & -6.414 & $<0.001$ \\
\hline CRNN & -6.334 & $<0.001$ \\
\hline MUC21 & -6.299 & $<0.001$ \\
\hline TARM1 & -6.022 & $<0.001$ \\
\hline MYOC & -6.006 & $<0.001$ \\
\hline SPANXD & 5.792 & $<0.001$ \\
\hline BAAT & 5.845 & $<0.001$ \\
\hline DPPA2 & 5.886 & $<0.001$ \\
\hline POU3F2 & 5.987 & $<0.001$ \\
\hline GAGE1 & 6.077 & $<0.001$ \\
\hline NEUROD4 & 6.205 & $<0.001$ \\
\hline GJA8 & 6.441 & $<0.001$ \\
\hline ELAVL3 & 6.553 & $<0.001$ \\
\hline GAD2 & 6.623 & $<0.001$ \\
\hline ASCL1 & 6.705 & $<0.001$ \\
\hline SOST & 6.779 & $<0.001$ \\
\hline FGF3 & 8.563 & $<0.001$ \\
\hline $\mathrm{GHRH}$ & 9.033 & $<0.001$ \\
\hline
\end{tabular}

FDR, false discovery rate.

ELISA was used to measure and compare the content of representative differential gene serum samples. All procedures performed in this study involving human participants were in accordance with the Declaration of Helsinki (as revised in 2013). The study was approved by the Ethics Committee of Hainan General Hospital, Hainan Affiliated Hospital of Hainan Medical University (No.: 20180A21) and informed consent was taken from all the patients.

\section{Statistical analysis}

This study used R software (V3.5.1) and related $\mathrm{R}$ packages for statistical analysis. The enumeration data is expressed in the form of mean \pm standard deviation, using independent sample $t$-test. $\mathrm{P}<0.05$ indicated statistical significance.

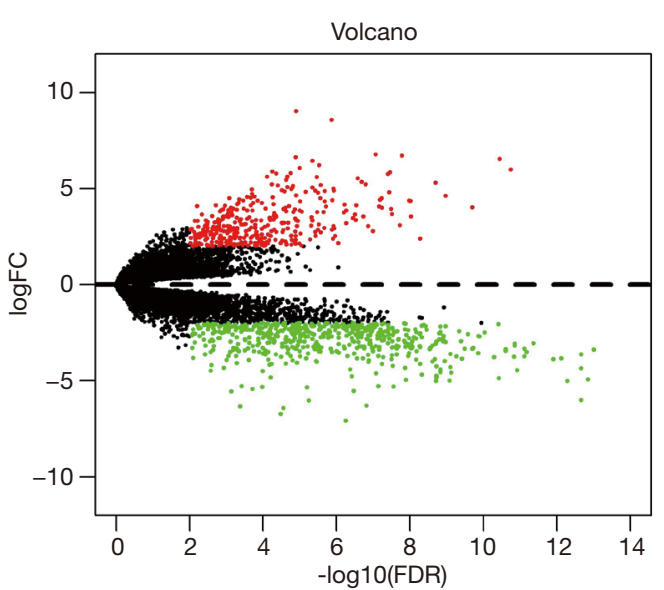

Figure 1 Map of differentially expressed genes. Red indicates upregulated genes in chronic heart failure $(\mathrm{CHF})$ tissue, and green indicates down-regulated genes in CHF tissue. The abscissa represents $\operatorname{lgFDR}$, and the ordinate represents $\log _{2} \mathrm{FC}$. The screening criteria were $\mid \log _{2} \mathrm{FCl}>2$ and false discovery rate (FDR) $<0.05$.

\section{Results}

\section{Differential gene screening}

Using $\mid \log _{2} \mathrm{FCl}>2$ and FDR $<0.05$ as screening conditions, a total of 902 differentially expressed genes between CHF and normal heart tissues were screened, including 354 upregulated genes in CHF and 548 down-regulated genes in CHF. According to the order of $\mid \log _{2} \mathrm{FCl}$ values, the top 20 differentially expressed immune-related genes with the most significant differences are shown in Table 1. Figure 1 illustrates the differentially expressed genes in $49 \mathrm{CHF}$ and 44 normal heart tissues.

\section{GO functional enrichment}

As a functional classification system, GO can be divided into 3 parts: molecular function (MF), biological process (BP), and cellular component (CC). In this study, the differentially expressed genes in the heart tissue of $\mathrm{CHF}$ patients were enriched by GO. There were $36 \mathrm{GO}$ terms with FDR $<0.05$, including 6 terms in MF, 12 terms in BP, and 18 terms in CC (Figure 2). After enhancing 70 genes with the most significant differential expression by GO, the results indicated a significant enrichment in the extracellular domain, positive regulation of neuronal differentiation, sequence-specific DNA binding, ion mode transport regulation, and activity of serotonin-activated selective- 


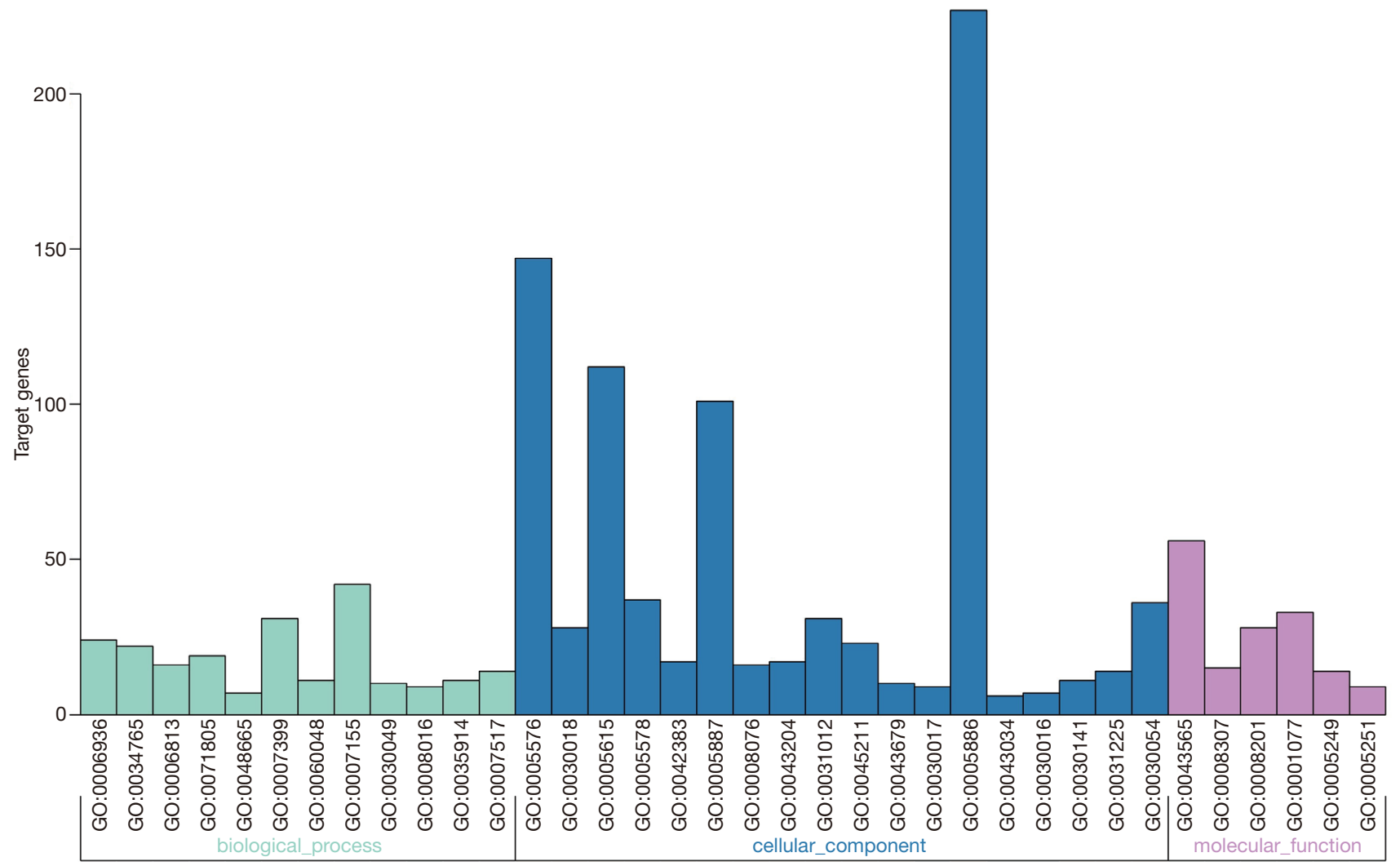

Figure 2 Gene Ontology (GO) enrichment analysis of differentially expressed genes. Green represents biological process (BP), blue represents cellular component (CC), and red represents molecular function (MF). The abscissa represents the GO terms, and the ordinate represents the number of target genes.

cation channels, as shown in Figure 3.

\section{KEGG patbway enrichment}

In this study, KEGG signaling pathway enrichment analysis of differentially expressed genes in CHF tissue and normal heart tissue was used to explore the signaling pathways of differential gene enrichment. The results showed that the differentially expressed genes were mainly enriched in neuroactive ligand-receptor interaction, calcium ion signaling pathway, vascular smooth muscle contraction, and other signaling pathways (Figure 4).

\section{Validation of representative differential gene serological samples}

By consulting the literature, the study used FGF3GHRH as a representative differential gene for serological sample verification. ELISA results showed that the expression level of GHRH in patients with heart failure was significantly higher than that in healthy subjects $(\mathrm{P}<0.05)$, as shown in Figure 5.

\section{Discussion}

CHF, defined as the lack of sufficient blood to meet the body's needs, is a syndrome associated with a high incidence rate and mortality. The 2 most common subtypes are ischemic heart disease and dilated cardiomyopathy. Once CHF occurs, it is irreversible. Clinical treatment focuses on reducing the load on the heart and improving ventricular compliance. Some end-stage patients need heart transplantation. Therefore, exploring the pathogenesis of $\mathrm{CHF}$ and finding potential drug targets to improve cardiac function and structure has been the focus of research.

Some researchers use animal models combined with functional genomics to study the molecular basis of CHF. Previous studies linked the genetic characteristics 


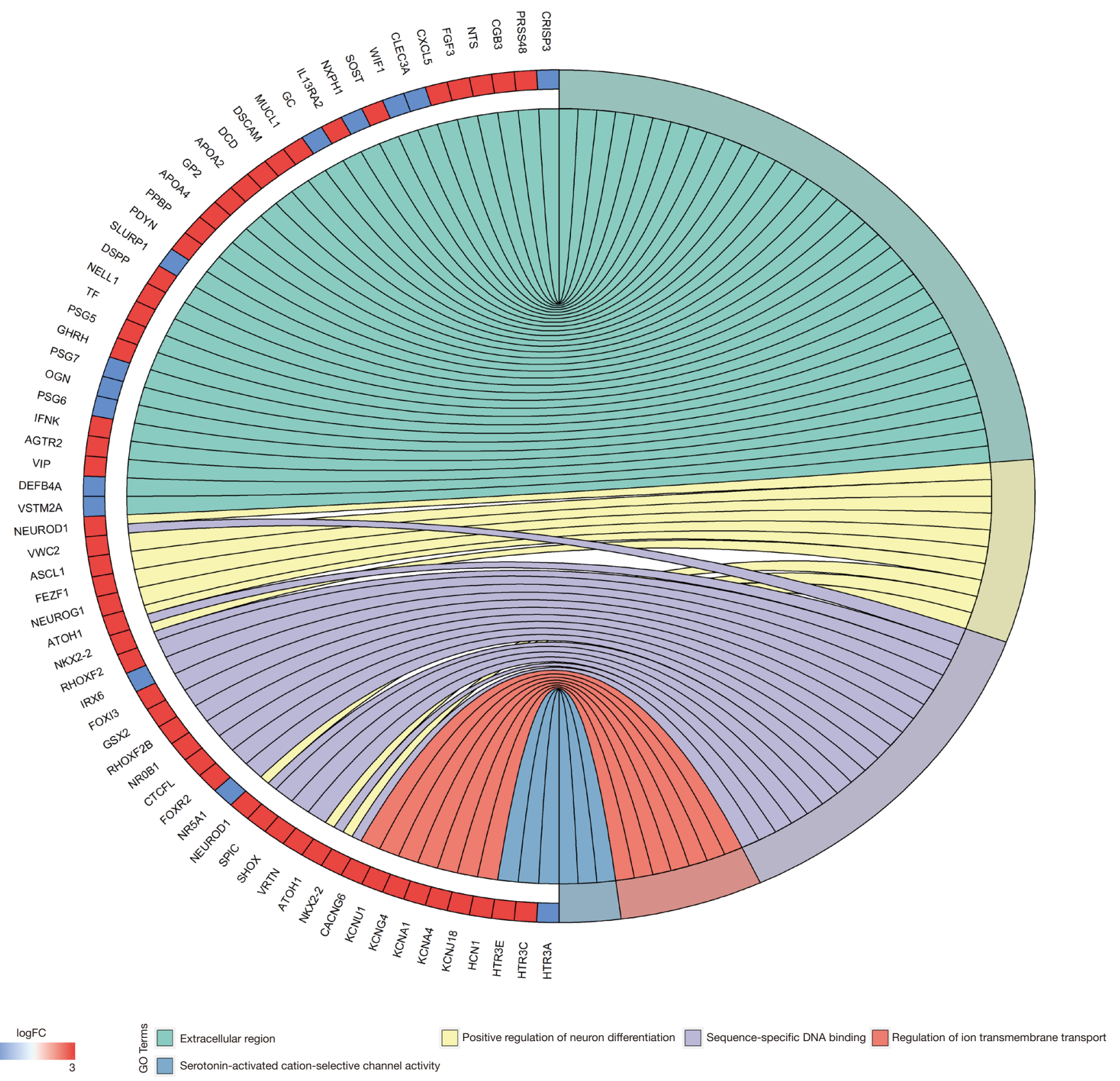

Figure 3 Genes with the most significant differential expression were enriched by Gene Ontology (GO). Blue indicates down-regulated genes, red indicates up-regulated genes.

of human blood with the results of CHF. Several recent studies utilized transcriptome sequencing of human cardiac tissue to explore their hypothesis. They found that endstage $\mathrm{CHF}$ was associated with increased gene expression levels of matrix/cytoskeleton and proteolysis/stress. Some studies used microarray and machine learning methods to distinguish patients with histological evidence of ischemic injury from patients without a history of myocardial infarction, revascularization, or coronary artery disease (14).

In recent years, the rapid development of full transcriptome sequencing technology has provided new opportunities to study disease pathogenesis at the molecular 


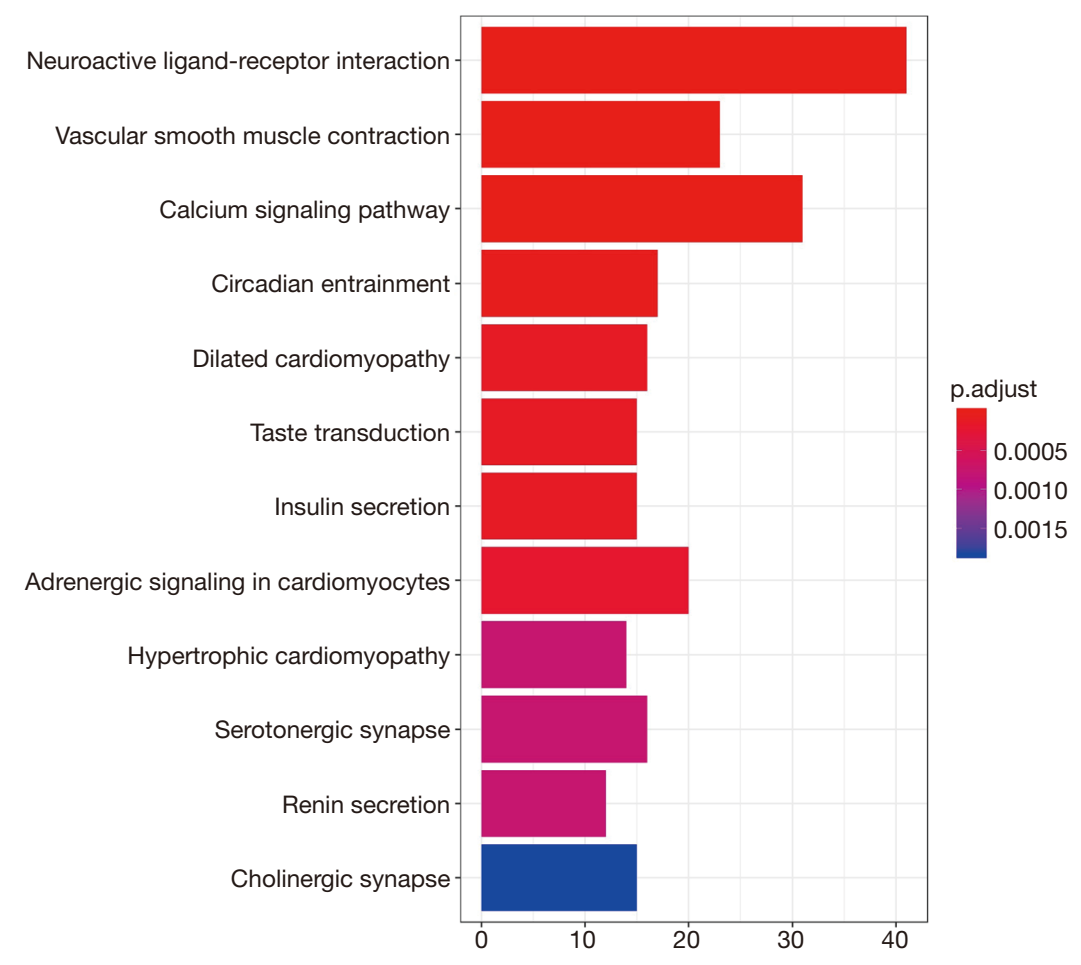

Figure 4 Kyoto Encyclopedia of Genes and Genomes (KEGG) pathway enrichment of the most significant genes. The abscissa represents the number of enriched genes, and the ordinate represents pathways. The color gradient from red to blue indicates $\mathrm{P}$ value changes from small to large after correction.

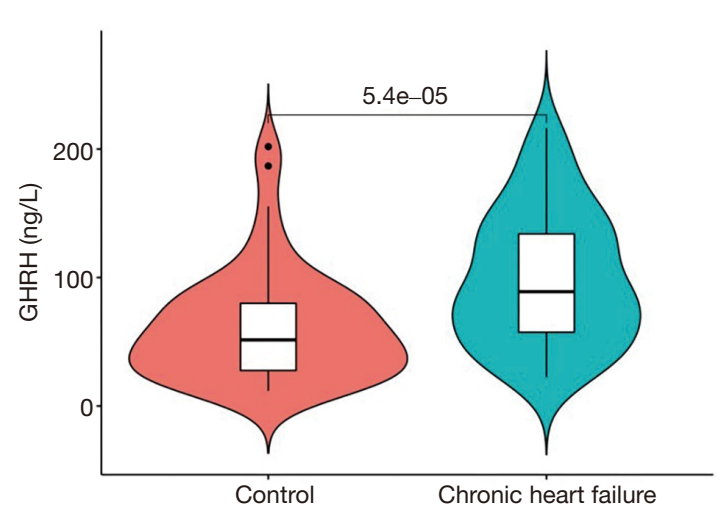

Figure 5 Comparison of serum levels of representative differential gene GHRH patients with heart failure and healthy subjects.

level. It greatly promotes the exploration and determination of molecular pathogenesis. Compared with previous studies, this study has some advantages. First, the sample size of previous studies was small. We used two public databases, GEO and GTEx, to expand the sample size, especially the content of normal samples, and to some extent compensate for the lack of tissue in normal heart samples. Second, the criteria for screening differential genes in this study are more stringent, and the differential expression multiples of differential genes are greater.

This study screened the differentially expressed genes between the heart tissues of patients with $\mathrm{CHF}$ and normal heart tissues. We also performed GO function and KEGG pathway enrichment analysis. In this study, the neuroactive ligand-receptor interaction pathway was the most abundant and statistically significant. Most of the ligands and receptors on the plasma membrane are concentrated in this pathway, which indicates that the pathogenesis of $\mathrm{CHF}$ may be closely related to intracellular and extracellular ion pathways and signal transduction. This study also found that the genes with the most significant differential expression were enriched in the calcium signaling pathway, which is considered to be closely related to cardiac arrhythmia, cardiac hypertrophy, and heart failure, and may be involved in the occurrence and progression of CHF (15). Studies have shown that the activation of the calcium signaling pathway in cardiomyocytes can lead to cardiac hypertrophy, 
further developing into CHF (16). This study also found that the vascular smooth muscle contraction pathway may play an essential role in the occurrence and progression of CHF. The vascular smooth muscle contraction pathway mediates hypertension, portal hypertension, and vascular endothelial injury $(17,18)$. These pathological changes increase the risk of CHF to a certain extent.

GHRH gene is closely related to the occurrence and progression of chronic heart failure. Studies have shown that GHRH gene has a protective effect on myocardial tissue after myocardial infarction (19). Studies have also shown that it can stimulate the self-renewal of cardiac stem cells and promote their survival (20). It is difficult to obtain heart tissue samples, and its clinical application mostly relies on serum samples. This study uses it as a representative differential gene to explore the difference in serum content between patients with heart failure and normal subjects. The results show that GHRH is highly expressed in patients with heart failure.

The main limitations of this study should be noted. First, the number of heart tissue samples of CHF was small and needs to be further expanded to confirm the significance of these results. Second, this study lacks in vitro experimental verification. We explored the differentially expressed genes and predicted the possible pathogenic mechanisms and signaling pathways. The data analysis of the secondgeneration sequencing results still needs to be confirmed by results from independent in vitro experiments and should also be further explored in regards to its clinical practicability.

In conclusion, there are 902 differentially expressed genes in CHF tissue, including those enriched in the extracellular domain, positive regulation of neuronal differentiation, sequence-specific DNA binding, ion mode transport regulation, and the activity of serotonin-activated selective-cation channels. The enriched genes were also associated with neuroactive ligand-receptor interaction, calcium signaling pathway, vascular smooth muscle contraction, and other related signaling pathways.

\section{Acknowledgments}

Funding: None.

\section{Footnote}

Reporting Checklist: The authors have completed the STREGA reporting checklist. Available at https://dx.doi. org/10.21037/apm-21-1854

Data Sharing Statement: Available at https://dx.doi. org/10.21037/apm-21-1854

Conflicts of Interest: All authors have completed the ICMJE uniform disclosure form (available at https://dx.doi. org/10.21037/apm-21-1854). The authors have no conflicts of interest to declare.

Ethical Statement: The authors are accountable for all aspects of the work in ensuring that questions related to the accuracy or integrity of any part of the work are appropriately investigated and resolved. All procedures performed in this study involving human participants were in accordance with the Declaration of Helsinki (as revised in 2013). The study was approved by the Ethics Committee of Hainan General Hospital, Hainan Affiliated Hospital of Hainan Medical University (No.: 20180A21) and informed consent was taken from all the patients.

Open Access Statement: This is an Open Access article distributed in accordance with the Creative Commons Attribution-NonCommercial-NoDerivs 4.0 International License (CC BY-NC-ND 4.0), which permits the noncommercial replication and distribution of the article with the strict proviso that no changes or edits are made and the original work is properly cited (including links to both the formal publication through the relevant DOI and the license). See: https://creativecommons.org/licenses/by-nc-nd/4.0/.

\section{References}

1. McMurray JJ, Pfeffer MA. Heart failure. Lancet 2005;365:1877-89.

2. Metra M, Teerlink JR. Heart failure. Lancet 2017;390:1981-95.

3. Hoffman TM. Chronic Heart Failure. Pediatr Crit Care Med 2016;17:S119-23.

4. Gedela M, Khan M, Jonsson O. Heart Failure. S D Med 2015;68:403-5, 407-9.

5. Huffman MD, Roth GA, Sliwa K, et al. editors. Cardiovascular, Respiratory, and Related Disorders. 3rd ed. Washington (DC): The International Bank for Reconstruction and Development/The World Bank; 2017 Nov 17. Chapter 10.

6. Baman JR, Ahmad FS. Heart Failure. JAMA 2020;324:1015. 
7. Liao J, Wang J, Liu Y, et al. Transcriptome sequencing of lncRNA, miRNA, mRNA and interaction network constructing in coronary heart disease. BMC Med Genomics 2019;12:124.

8. Zhang H, He L, Cai L. Transcriptome Sequencing: RNASeq. Methods Mol Biol 2018;1754:15-27.

9. Hua X, Wang YY, Jia P, et al. Multi-level transcriptome sequencing identifies COL1A1 as a candidate marker in human heart failure progression. BMC Med 2020;18:2 .

10. Li H, Yang HH, Sun ZG, et al. Whole-transcriptome sequencing of knee joint cartilage from osteoarthritis patients. Bone Joint Res 2019;8:288-301.

11. Zhang NN, Lin T, Xiao M, et al. Transcriptome sequencing analysis of mono-ADP-ribosylation in colorectal cancer cells. Oncol Rep 2020;43:1413-28.

12. Zhang J, Huang JY, Chen YN, et al. Whole genome and transcriptome sequencing of matched primary and peritoneal metastatic gastric carcinoma. Sci Rep 2015;5:13750.

13. Zhao J, Lv T, Quan J, et al. Identification of target genes in cardiomyopathy with fibrosis and cardiac remodeling. J Biomed Sci 2018;25:63.

14. Meder B, Haas J, Sedaghat-Hamedani F, et al. EpigenomeWide Association Study Identifies Cardiac Gene Patterning and a Novel Class of Biomarkers for Heart Failure. Circulation 2017;136:1528-44.

Cite this article as: Fang X, Zeng X, Wang M, Qin L, Tan C, Wu J. Enrichment analysis of differentially expressed genes in chronic heart failure. Ann Palliat Med 2021;10(8):9049-9056. doi: 10.21037/apm-21-1854
15. Wang $X$, Wang $M$, Wang $W$, et al. Transcriptional changes of Pacific oyster Crassostrea gigas reveal essential role of calcium signal pathway in response to $\mathrm{CO} 2$-driven acidification. Sci Total Environ 2020;741:140177.

16. Ma Y, Yue Z, Zhang B, et al. Calcium Signal Pathway is Involved in Prostaglandin E2 Induced Cardiac Fibrosis in Cardiac Fibroblasts. J Pharm Pharm Sci 2018;21:326-39.

17. Birulina YG, Ivanov VV, Buyko EE, et al. Regulation of Vascular Smooth Muscle Contractions in the Model of Metabolic Syndrome. Bull Exp Biol Med 2020;170:196-9.

18. Yang GM, Yan K, Wang P, et al. ITRAQ-Based Proteomics Analysis Reveals the Effect of Neoliensinine on KCl-Induced Vascular Smooth Muscle Contraction by Inhibiting Regulatory Light Chain Phosphorylation. Front Pharmacol 2019;10:979.

19. Kanashiro-Takeuchi RM, Tziomalos K, Takeuchi LM, et al. Cardioprotective effects of growth hormone-releasing hormone agonist after myocardial infarction. Proc Natl Acad Sci U S A 2010;107:2604-9.

20. Florea V, Majid SS, Kanashiro-Takeuchi RM, et al. Agonists of growth hormone-releasing hormone stimulate self-renewal of cardiac stem cells and promote their survival. Proc Natl Acad Sci U S A 2014;111:17260-5.

(English Language Editor: C. Beltar) 